\title{
Refractory Status Epilepticus Following Ingestion of Organochlorine Pesticide Endosulphan- A Case Report
}

\author{
Bhattarai $S,{ }^{1^{*}}$ Kohli $S C^{1}$
}

${ }^{1}$ Department of Medicine, Manipal College of Medical sciences, Pokhara, Nepal

\begin{abstract}
* Corresponding Author:
Dr Subash Bhattarai, MBBS, MD

Manipal Teaching hospital, Pokhara, Nepal.

Email: kiwisubash@yahoo.com

\section{Citation}

Bhattarai S, Kohli SC. Refractory status epilepticus following ingestion of organochlorine pesticide endosulphan - a case report. Nepal Journal of Medical Sciences. 2012; 1(1): 59-61.
\end{abstract}

\begin{abstract}
A 22 years old female was brought to emergency department of Manipal teaching hospital, Pokhara, Nepal with suicidal consumption of 35 grams of endosulphan .She presented with status epilepticus not responding to intravenous benzodiazepine, loading doses of phenytoin and phenobarbitone and was managed by intravenous propofol and subsequently with continuous infusion of midazolam. Refractory status epilepticus is a common problem following ingestion of endosulphan and unless aggressively treated, it is associated with high morbidity and mortality. Endosulphan poisoning is often completely reversible with immediate and appropriate treatment.
\end{abstract}

Keywords: Endosulphan poisoning; seizure; refractory status epilepticus; benzodiazepine; propofol.

\section{Introduction:}

Status epilepticus (SE) is a medical emergency. SE is defined clinically as seizure lasting for more than 5 minutes, or 2 or more discrete seizures without recovery of consciousness in between. ${ }^{1} \mathrm{SE}$ may progress to refractory status epilepticus (RSE) which has been described as SE refractory to 2-3 first line anticonvulsants, and/or SE persisting for up to 2 hours. ${ }^{2}$

Endosulphan-induced RSE is an important cause of seizures and deaths in South Asia. The management of RSE is challenging. With emergent management and treatment, endosulphan poisoning is completely reversible. Salient features of endosulphan toxicity and its management is discussed.

\section{Case report:}

A previously well, 22-year-old female weighing $51 \mathrm{~kg}$ was brought by her relatives in the emergency department of Manipal Teaching Hospital, a tertiary care hospital after being found lying in an unconscious state .An offensive odour and froathing from mouth was noticed alongside a $100 \mathrm{ml}$ of emptied bottle containing pesticide labeled THIODAN endosulphan $(35 \mathrm{~g} / 100 \mathrm{ml})$ lying by her side. The history suggested that she had probably consumed 100 $\mathrm{ml}$ of endosulphan approximately about 90 minutes before reaching to the emergency department.

In the emergency department, generalized tonic-clonic seizures were observed, initially lasting for 1-2 minutes every 3-5 minutes without regaining consciousness in between. Patient's vital parameters showed tachypnoea with respiratory rate of 36 per minute, tachycardia with heart rate of 110 per minute; was afebrile and had blood pressure of 140/90 $\mathrm{mm}$ of $\mathrm{Hg}$. In between the seizures, patient had glassglow coma scale (GCS) of 8/15.On examination, the patient had bilateral mid dilated pupils reacting to light along with hyperreflexia, clonus and bilateral extensor planter response. There was no focal neurological deficit. Diffuse crepitations were heard all over the chest and heart sounds were normal. 
Gastric lavage was done with activated charcoal. Airway was secured and oxygen was given via mask. Patient was given $5 \mathrm{mg}$ of intravenous lorazepam over $1 \mathrm{~min}$ and same dose was repeated after 5 minutes due to lack of response. Despite the repeated dose of intravenous (IV) lorazepam, the patient continued to have status epilepticus. A loading dose of $1000 \mathrm{mg}$ of injection phenytoin in $100 \mathrm{ml}$ of normal saline intravenously over 20 minutes was given but it failed to control seizure. A repeat dose of injection phenytoin $500 \mathrm{mg}$ IV too was also ineffective to control convulsions. Thereafter, a loading dose of injection phenobarbitone 1000 $\mathrm{mg}$ intravenously was given which resulted in temporary cessation of convulsions. The convulsions reappeared after 10 minutes.

In view of failure of above medications to control seizures, further management as for refractory status epilepticus was initiated. Patient was intubated and transferred to intensive care unit (ICU). Patient was put on mechanical ventilation and a loading dose of injection Propofol $160 \mathrm{mg}$ intravenously was administered and was followed by continuous infusion at the rate of $240 \mathrm{mg}$ per hour. With this, the seizures were controlled. The patient remained seizure free for about 20 minutes but they reappeared thereafter. A bolus injection of $200 \mathrm{mg}$ of thiopentone infused intravenously controlled the seizure. There were further no episodes of convulsions.

Patient remained seizure free for the next 24 hours with propofol infusion. It was tapered in next 24 hours and was replaced by midazolam infusion IV initially at the rate of 2 $\mathrm{mg} /$ hour for 24 hours and tapered off after 48 hours. On $4^{\text {th }}$ day patient was taken off from the ventilator as the patient maintained spontaneous respiration and oxygen saturation. Patient GCS was 15/15 and was extubated successfully. Continuous infusion of antiepileptic was replaced by Phenytoin orally $300 \mathrm{mg}$ once daily.

The results of basic investigations were: serum glucose, sodium, potassium, calcium and liver function test were found to be within normal limit. Computed tomography of head done after cessation of seizure on fifth day was normal.

Patient was kept under observation in ICU for next $24 \mathrm{hrs}$ and was later transferred to general ward. Patient remained symptom free thereafter and was finally discharged after 5 days.

\section{Discussion:}

Endosulphan is a polychlorinated hydrocarbon insecticide used in agriculture. The predominant toxicological effect is overstimulation of central nervous system by inhibiting $\mathrm{Ca}^{++}$ and $\mathrm{Mg}^{++}$ATP ase and antagonizing chloride ion transport in gamma aminobutyric acid (GABA) receptors. ${ }^{3}$ The clinical signs include seizure, nausea, vomiting, abdominal discomfort, hyperaesthesia of the mouth, tongue face, and extremities, headaches, agitation, hyperactivity, tremor, ataxia, incoordination, confusion, dizziness, syncope, myoclonus and respiratory depression., ${ }^{1,3}$ Convulsions are common and severe manifestations. ${ }^{3}$ Endosulphan is also toxic to liver, kidneys and lungs and can cause rhabdomyolysis in higher doses. ${ }^{4}$ Hypoxia may be secondary to aspiration of vomitus or respiratory failure. Refractory status epilepticus is a common problem following ingestion of endosulphan and unless aggressively treated, it is associated with high morbidity and mortality. Endosulphan poisoning is often completely reversible with immediate and appropriate treatment. Approximately $80 \%$ of deaths from endosulphan poisoning occur within $24 \mathrm{~h}$ of presentation, the majority within six hours. Most deaths are secondary to respiratory depression, arrhythmia and cardio respiratory arrest amidst ongoing seizures. ${ }^{4}$

Roberts DM et $\mathrm{al}^{5}$ reported a fatal case of refractory status epilepticus presenting to a rural general hospital in Sri Lanka after intentional self-poisoning with the organochlorine insecticide endosulphan. Kartas et al, ${ }^{6}$ reported 23 cases of endosulphan poisoning out of which 19 developed refractory seizures. Jeong $\mathrm{M} \mathrm{M}$ et $\mathrm{al}^{7}$ in South Korea reported fifty-two patients who presented with acute endosulphan poisoning between January 2001 and January 2007 . Sixteen (30.7\%) of the 52 patients died, and 48 patients experienced seizures. Refractory status epilepticus was the most common cause of death in this series $(75.0 \%)$. Amount ingested being greater than 35 grams of endosulphan was the most found to be an independent variable that predicted patient mortality.

Management of SE requires early identification and treatment. ${ }^{1}$ Management of seizures along with gastric lavage and maintenance of ABC (airway, breathing, circulation) is the priority after endosulphan poisoning. Intravenous administration of a benzodiazepine, followed by phenytoin and/or phenobarbitone is first-line treatment of SE. Other agents viz, propofol, midazolam, lignocaine,valproate, ketamine, and inhalational anaesthesia may be considered if seizures continue Refractory status epilepticus that has not responded to hydantoins and/or phenobarbitone requires general anaesthesia using either thiopental (thiopentone) or propofol. $^{8}$

Seizure duration greater than one hour is associated with an extremely poor outcome. It has been recommended that general anaesthesia be induced where SE continues beyond 60-90 minutes, despite adequate administration of anticonvulsants. However, where resources permit, it is reasonable to initiate full anaesthesia before one hour, and probably after 30 minutes. Prompt and adequate therapy may prevent progression to RSE, or curtail established RSE. ${ }^{9}$ 
High-dose benzodiazepines, especially midazolam form the cornerstone of RSE treatment in many centers. ${ }^{10}$ Propofol with its rapid onset and offset of anesthetic action made it seem the ideal drug for critical care use. ${ }^{11}$ Aggarwal $\mathrm{P}$ et $\mathrm{al}^{12}$ reported four cases with status epilepticus treated with intravenous lidocaine who had failed to respond to diazepam and phenytoin therapy. Two cases responded to a single dose of lidocaine, one required a second dose of lidocaine to control seizures, and the fourth patient failed to respond and died as a result of associated severe head injury. Jain et $\mathrm{al}^{13}$ reported a case of suicidal consumption of endosulphan, presented with refractory status epilepticus successfully treated with injection levetiracetam intravenously.

Young et $\mathrm{al}^{14}$ reported seven patients treated with isoflurane or desflurane, four of whom had good outcomes. Ketamine is also useful in RSE treatment because its intrinsic sympathomimetic properties cause it to raise systemic arterial pressure. In a series of 7 RSE patients, 5 experienced this beneficial effect, and only one had a further decline in blood pressure. ${ }^{15}$ Anticonvulsant drugs like topiramate has also been found beneficial in management of RSE. ${ }^{16}$

\section{Conclusion}

Endosulphan poisoning is an important cause of RSE in developing countries in South Asia like Nepal, India and Sri Lanka Few resources list pesticides such as endosulphan as important causes of SE. RSE remains difficult to treat though many potentially useful agents are available. Randomized trials would help in formulating guidelines for early prediction of complications due to endosulphan poisoning and management of RSE, and reduce morbidity and mortality.

\section{References}

1. Lowenstein DH. Status epilepticus: an overview of the clinical problem, Epilepsia 1999;40:53-8.

2. Mayer SA, Claassen J, Lokin J, et al. Refractory status epilepticus: frequency, risk factors, and impact on outcome, Arch. Neurol. 20002;59:205-10.

3. Holland MG. Insecticides: organochlorines, pyrethrins, and deet. In: Goldfrank LR, Flomenbaum NE, Lewin NA (eds).Goldfrank's Toxicological Emergencies, 7th edn. McGraw-Hill, New York, NY, 2002;1336-78.

4. Eyer F, Felgenhauer N, Jetzinger E, et al. Acute endosulphan poisoning with cerebral edema and cardiac failure. J Toxicol Clin Toxicol 2004;42:927-30.
5. Roberts DM, Dissanayake W, Sheriff HR, et al. Refractory status epilepticus following self-poisoning with the organochlorine pesticide endosulphan. J Clin Neurosci 2004;11:760-2.

6. Karatas AD, Aygun D, Baydin A. Characteristics of endosulphan poisoning: a study of 23 cases. Singapore Med J 2006;47:1030-2.

7. Jeong $\mathrm{M}$ M, Byeong $\mathrm{J}$ C. Acute endosulphan poisoning: a retrospective study. Hum Exp Toxicol 2009;28:309-16.

8. Claassen J, Hirsch L, Emerson R, et al. Treatment of refractory status epilepticus with pentobarbital, propofol, or midazolam: a systematic review, Epilepsia 2002;43:146-53.

9. Martin Holtkamp. Treatment strategies for refractory status epilepticus. Current Opinion in Critical Care 2011, 17:94-100.

10. Prasad A, Worrall BB, Bertram EH, et al. Propofol and midazolam in the treatment of refractory status epilepticus. Epilepsia 2001;42:380 - 6.

11. Stecker MM, Kramer TH, Raps EC, et al. Treatment of refractory status epilepticus with propofol: clinical and pharmacokinetic findings. Epilepsia 1998;39:18-26.

12. Aggarwal P, Wail JP. Lidocaine in refractory status epilepticus: A forgotten drug in the emergency department. Am J Emerg Med 1993;11:243-4.

13. Jain P. K., Sharma A K, Agarwal N et al. Management of refractory status epilepticus in acute endosulphan poisoning by levetiracetam: A case report. Journal of Clinical Medicine and Research 2011;3:57-9.

14. Young GB, Mirsattari SM, Sharpe MD. Treatment of refractory status epilepticus with inhalational anesthetic agents isoflurane and desflurane. Arch Neurol 2004;61:1254-9.

15. Nathan BN, Smith TL, Bleck TP. The use of ketamine in refractory status epilepticus. Neurology 2002;58:197.

16. Tarulli A, Drislane FW.The use of topiramate in refractory status epilepticus. Neurology 2004;62:837. 